\title{
Age-related Effects of Heroin on Gene Expression in the Hippocampus and Striatum of Cynomolgus Monkeys
}

\author{
Mi Ran Choi ${ }^{1, *}$, Yeung-Bae Jin ${ }^{2, *}$, Sol Hee Bang ${ }^{1}$, Chang-Nim Im ${ }^{1}$, Youngjeon Lee ${ }^{2}$, Han-Na Kim ${ }^{2}$, \\ Kyu-Tae Chang ${ }^{2,3}$, Sang-Rae Lee ${ }^{2,3}$, Dai-Jin Kim ${ }^{1}$ \\ ${ }^{1}$ Department of Psychiatry, Seoul St. Mary's Hospital, College of Medicine, The Catholic University of Korea, Seoul, ${ }^{2}$ National Primate Research \\ Center (NPRC), Korea Research Institute of Bioscience and Biotechnology (KRIBB), Cheongju, ${ }^{3}$ Department of Functional Genomics, University \\ of Science and Technology, Daejeon, Korea
}

\begin{abstract}
Objective: The aim of this study was to investigate differentially expressed genes and their functions in the hippocampus and striatum after heroin administration in cynomolgus macaques of different ages.

Methods: Cynomolgus monkeys were divided by age as follows: 1 year (A1, $\mathrm{n}=2$ ); 3 to 4 years (A2, $\mathrm{n}=2$ ); 6 to 8 years $(\mathrm{A} 3, \mathrm{n}=2)$; and older than 11 years $(\mathrm{A} 4, \mathrm{n}=2)$. After heroin was injected intramuscularly into the monkeys $(0.6 \mathrm{mg} / \mathrm{kg})$, we performed large-scale transcriptome profiling in the hippocampus $(\mathrm{H})$ and striatum $(\mathrm{S})$ using RNA sequencing technology. Some genes were validated with real-time quantitative PCR.

Results: In the hippocampus, the gene expression of $\mathrm{A} 1 \mathrm{H}$ was similar to that of $\mathrm{A} 4 \mathrm{H}$, while the gene expression of $\mathrm{A} 2 \mathrm{H}$ was similar to that of $\mathrm{A} 3 \mathrm{H}$. Genes associated with the mitogen-activated protein kinase signaling pathway (STMN1, FGF14, and MAPT) and $\gamma$-aminobutyric acid-ergic synapses (GABBR2 and GAD1) were differentially expressed among control and heroin-treated animals. Differential gene expression between A1S and A4S was the least significant, while differential gene expression between $\mathrm{A} 3 \mathrm{~S}$ and $\mathrm{A} 2 \mathrm{~S}$ was the most significant. Genes associated with the neurotrophin signaling pathway (NTRK1 and NGFR), autophagy (ATG5), and dopaminergic synapses ( $A K T 1)$ in the striatum were differentially expressed among control and heroin-treated animals.

Conclusion: These results suggest that even a single heroin exposure can cause differential gene expression in the hippocampus and striatum of nonhuman primates at different ages.
\end{abstract}

KEY WORDS: Age factors; Heroin; Hippocampus; Nonhuman primates; Striatum; Gene expression profiling.

\section{INTRODUCTION}

Heroin is an opioid, interacting with receptors on neuronal cells in the brain to allow euphoric effects and pain relief [1]. Heroin is an illicit drug, unlike other opioids that

Received: February 27, 2019 / Revised: May 2, 2019

Accepted: May 3, 2019

Address for correspondence: Dai-Jin Kim

Department of Psychiatry, Seoul St. Mary's Hospital, College of

Medicine, The Catholic University of Korea, 222 Banpo-daero,

Seocho-gu, Seoul 06591, Korea

E-mail: kdj922@catholic.ac.kr

ORCID: https://orcid.org/0000-0001-9408-5639

Sang-Rae Lee

National Primate Research Center (NPRC), Korea Research Institute of Bioscience and Biotechnology (KRIBB), 30 Yeonggudanji-ro,

Ochang-eup, Cheongwon-gu, Cheongju 28116, Korea

E-mail: srlee@kribb.re.kr

ORCID: https://orcid.org/0000-0001-8400-5973

*These authors contributed equally to this study as co-first authors. can be purchased by prescription. Heroin use has increased across most age groups in the United States, especially ages 18 to 25 years. This increased use has elevated the possibility of heroin abuse leading to death [1]. Asia is currently the biggest market for heroin; two-thirds of people who abuse opioids (including heroin) live in Asia [2]. Previous study has shown that heroin abuse causes deleterious effects on cognitive function and impulse control [3].

The hippocampus, where neurogenesis occurs, is responsible for learning, memory, and spatial cognition [4]. Therefore, chronic exposure to heroin can destroy learning, memory, and cognitive function [5]. Researchers have demonstrated that acute heroin exposure negatively impacts attention, motivation, and impulse control in mice, suggesting that even short-term exposure affects various functions in the brain $[6,7]$. In addition, mice that experience prenatal heroin exposure show impairment of learn-

(ㄷ) This is an Open-Access article distributed under the terms of the Creative Commons Attribution Non-Commercial License (http://creativecommons.org/licenses/by-nc/4.0) which permits unrestricted non-commercial use, distribution, and reproduction in any medium, provided the original work is properly cited. 
ing and memory. These mice display increased neuronal apoptosis and alterations of protein kinase $\mathrm{C}$ activity in the hippocampus, indicating that indirect exposure to heroin negatively affects the brain [8]. In a study by Benoilid et al. [9], a single heroin inhalation caused cortical laminar necrosis in the hippocampus, as well as amnesia. However, the underlying mechanisms remain uncertain.

The striatum is composed of the caudate nucleus, putamen, and nucleus accumbens (NAc). As a core motor control center, the striatum undergoes cellular and molecular adaptations on exposure to addictive drugs [10]. Acute administration of heroin induces a rapid increase of dopamine in the striatum of animal models [11]. In contrast, the chronic use of heroin reduces the level of dopamine transporter in the striatum of humans [12]. Chronic use also impairs transcriptional events associated with glutamatergic neurotransmission in the striatum of heroin addicts [13]. The size of the NAc in heroin-dependent patients is decreased compared to that of healthy controls, indicating that alterations in apoptosis or neurotoxicity may affect volumetric changes [14]. In humans, it is difficult to analyze molecular mechanisms in areas of the brain exposed to heroin, aside from postmortem analyses. For this reason, the effects of addictive drugs are often studied by analyzing drug-induced cellular and molecular changes in the brains of animals similar to humans.

The genome of the long-tailed cynomolgus macaque (Macaca fascicularis) shows 92.8\% similarity to humans [15]. In particular, their mesocortical dopaminergic system, neuroanatomical structures, neural circuits, and aging processes have many similarities to those of humans [16]. Based on these similarities, macaques have been used to study the molecular mechanisms associated with neurodegenerative diseases and drug abuse in the human brain $[17,18]$. To investigate the age-related effects of a single exposure to heroin on gene expression in the hippocampus and striatum, we injected heroin $(0.6 \mathrm{mg} / \mathrm{kg})$ into cynomolgus monkeys, performed large-scale transcriptome profiling using RNA sequencing (RNA-Seq), and analyzed certain functional regulatory networks. Based on our findings, we validated the messenger RNA (mRNA) expression levels of genes related to the mitogen-activated protein kinase (MAPK) signaling pathway, $\gamma$-aminobutyric acid (GABA)ergic synapses, and retrograde endocannabinoid signaling in the hippocampus. We further validated the mRNA expression levels of genes related to the neurotrophin signaling pathway, autophagy, oxytocin signaling pathway, and dopaminergic synapses in the striatum.

\section{METHODS}

\section{Animals}

Ten female cynomolgus monkeys with no history of previous participation in drug studies were used. On the basis of lifespan data for caged monkeys, we used a ratio of 1:3 when comparing ages of cynomolgus monkeys to humans [19]. The monkeys originated from Suzhou Xishan Zhongke Laboratory Animal Co. (Suzhou, China). They were maintained in individual indoor cages at the National Primate Research Center at the Korea Research Institute of Bioscience and Biotechnology (KRIBB), as described previously [20]. All procedures were approved by the KRIBB Institutional Animal Care and Use Committee (approval No., KRIBB-AEC-17079).

\section{Heroin Treatment}

Diamorphine (Johnson Matthey Macfarlan Smith, Edinburgh, Scotland) was freshly dissolved in $0.9 \%$ saline immediately before administration. Cynomolgus monkeys for heroin treatment were grouped by age as follows: 1 year (A1), 3 to 4 years (A2), 6 to 8 years (A3), and older than 11 years (A4). Ten monkeys were divided into a control group $(n=2)$ and four heroin-treated groups $(A 1, n=$ $2 ; \mathrm{A} 2, \mathrm{n}=2 ; \mathrm{A} 3, \mathrm{n}=2 ; \mathrm{A} 4, \mathrm{n}=2$ ). The control group received an intramuscular injection of $0.1 \mathrm{ml}$ of $0.9 \%$ saline. The heroin groups received a single-dose intramuscular injection of heroin $(0.6 \mathrm{mg} / \mathrm{kg})$ at 10:00 AM. The dosage for heroin administration was determined based on previous research [21]. Control and heroin-injected groups were sacrificed after they were maintained for 4 weeks to investigate whether a single injection of heroin had an effect on brain impairments even after a certain period of time.

\section{RNA-Seq Library Preparation and Sequencing}

Total RNA was extracted from the hippocampus and striatum in control $(n=1), A 1(n=1), A 2(n=1), A 3(n=$ $1)$, and $A 4(n=1)$ animals using TRIzol (Life Technologies, Carlsbad, CA, USA), according to the manufacturer's instructions. Total RNA integrity was assessed using a 2100 Bioanalyzer (Agilent Technologies, Palo Alto, CA, 
USA) and an RNA integrity number value greater than 8 . For RNA-Seq, RNA libraries were prepared with the TruSeq RNA library preparation kit (Illumina, San Diego, CA, USA) using a modified protocol. Briefly, mRNA was purified using oligo-dT-attached magnetic beads. The purified mRNA was cleaved and reverse-transcribed into first-strand complementary DNA (cDNA) using reverse transcriptase and random primers. The RNA template was removed, followed by second-strand cDNA synthesis. End repair, 3' adenylation, and adapter ligation were performed. The purified cDNA templates were then enriched using polymerase chain reaction (PCR). The constructed libraries were 101-bp paired-end sequenced using an Illumina HiSeq 2500 sequencer.

\section{Differential Gene Expression Analysis}

Raw reads obtained from RNA-Seq underwent quality control analyses using FastQC (version 0.10.1; http://www.bioinformatics, babraham.ac.uk/projects/fastqc/). To remove low-quality data and artifacts (including adaptor sequences, contaminant DNA, and PCR duplicates), preprocessing of reads was performed using Trimmomatic version 0.32 [22]. The preprocessed reads were mapped into a reference genome using TopHat software (version 2.1.0), and aligned reads were produced. The transcripts of each sample were assembled by Cufflinks [23] based on the fragments per kilobase of transcript per million mapped reads (FPKM) method. If there was a transcript with 0 FPKM in more than one of five samples per brain region (hippocampus or striatum), the transcript was excluded from the following analysis. To facilitate the log2 transformation, 1 was added to each FPKM value of the filtered genes. Filtered data were log2-transformed and subjected to quantile normalization. Statistical significance of the differential expression data was determined using an independent $t$ test and fold change (FC), in which the null hypothesis stated there are no differences among groups. The false discovery rate was controlled by adjusting the $p$ value using the Benjamini-Hochberg algorithm. For the differentially expressed gene (DEG) set, hierarchical clustering analysis was performed using complete linkage and Euclidean distance as a measure of similarity for the expression patterns of differentially expressed transcripts, while satisfying $|\mathrm{fc}| \geq 2$ and independent $t$ test raw $p<$ 0.05 . All data and visualization of DEGs were conducted using R 3.1.2 (www.r-project.org).

\section{Functional Annotation and Pathway Analysis}

The DAVID 6.8 tool (https://david.ncifcrf.gov) was used for functional annotation and gene enrichment of transcripts that were differentially expressed in response to heroin. Statistically over-represented gene ontology (GO) categories at $p<0.05$ were considered significant. GO categories were classified into three subcategories: biological process (BP), cellular component, and molecular function. After subsequently subdividing the categories into hyperlinked GO categories, we selected GO terms that showed a value of $p<0.05$. To analyze the biological responses and various canonical pathways associated with DEGs, the KEGG pathway database (http://www.kegg.jp/kegg/pathway.html) was employed. The KEGG pathway analysis produced molecular and cellular networks that might involve DEGs based on previously known associations between genes or proteins, if they are otherwise independent of established canonical pathways.

\section{Real-time Quantitative PCR (RT-qPCR)}

To validate the genes that were differentially expressed in RNA-Seq, we performed RT-qPCR. Total RNA extracted from the hippocampus and striatum of controls $(n=$ 2) and heroin-treated animals ( $A 1, n=2 ; A 2, n=2 ; A 3, n=$ 2; $A 4, n=2$ ) was reverse transcribed to cDNA using the Superscript RT III system (Thermo Fisher Scientific, San Jose, CA, USA). Details of the methods for QPCR have been described previously [20]. Experiments for qPCR were performed three times independently for all samples in each group (control, A1, A2, A3, and A4) to guarantee reliable results. The differential expression of genes in each sample was normalized to GAPDHexpression. The relative expression differences among control and heroin-treated groups were calculated using the $2^{-\Delta \Delta C T}$ method [24]. The primers used for the amplification of candidate genes are presented in Supplementary Table 1.

\section{Statistical Analysis}

All data obtained from qPCR are expressed as mean \pm standard error of the mean. The statistical significance of differences among groups was analyzed by one-way analysis of variance (ANOVA) using SPSS software (ver. 18.0; SPSS Inc., Chicago, IL, USA). ANOVA results (followed by Tukey's honestly significant difference post-hoc test) with $p<0.05$ were considered statistically significant. 


\section{RESULTS}

\section{Identification of Genes Expressed Differentially according to Age on Exposure to Heroin}

We investigated DEGs in the hippocampus $(\mathrm{H})$ and striatum (S) using RNA-Seq technology after exposing the monkeys to heroin. Control animals (ConH and ConS) were not treated with heroin. We extracted 33,886 and 30,513 transcripts in the hippocampus and striatum, respectively. We mined the data from the control and heroin-treated groups for each brain region (hippocampus and striatum) using a $\log 2$ fc cutoff of 1.5 ( $\mid f_{\mathrm{fc}} \geq 2$ ). We constructed heat maps of 6,540 transcripts in the hippocampus and 8,708 transcripts in the striatum. These transcripts satisfied the $\mid \mathrm{fcl} \geq 2$ conditions in at least one of the total comparison pairs. Heat maps were constructed by two-way hierarchical clustering based on similarities of expression (Fig. 1A, 1B). In the hippocampus, the gene expression pattern of $\mathrm{A} 1 \mathrm{H}$ was similar to that in $\mathrm{A} 4 \mathrm{H}$, while the gene expression pattern of $\mathrm{A} 2 \mathrm{H}$ was similar to that in $\mathrm{A} 3 \mathrm{H}$. Of the 6,540 transcripts in the hippocampus, $5,534,2,879,3,544$, and 4,990 transcripts were up-/

\section{A}

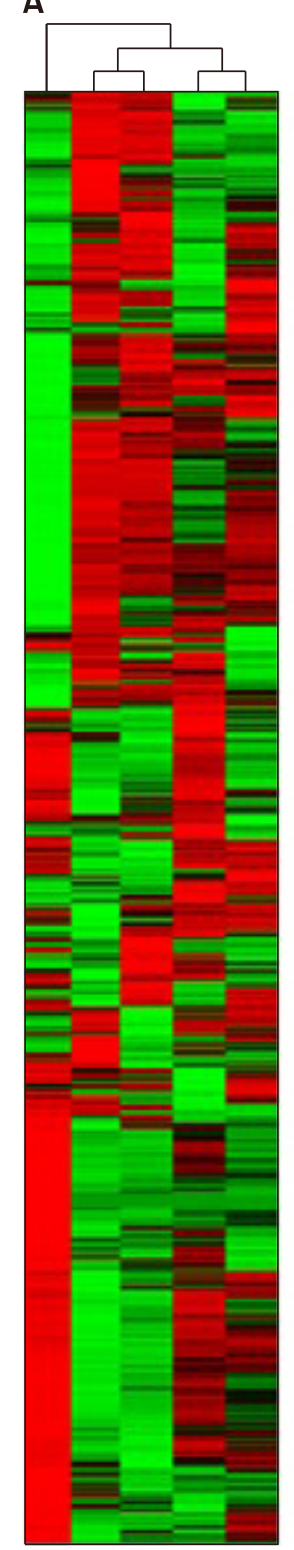

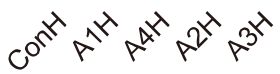

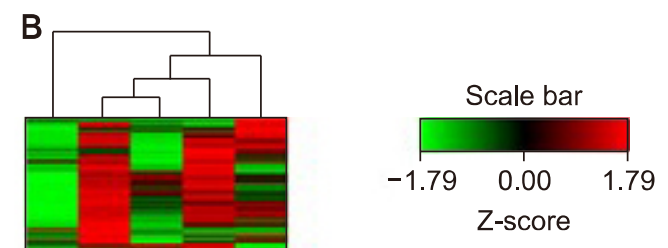

C

\begin{tabular}{|l||l||l||c|}
\hline \multicolumn{2}{|c||}{ Hippocampus } & \multicolumn{2}{c|}{ Striatum } \\
\hline A1H vs. ConH & 5,534 & A1S vs. ConS & 7,382 \\
\hline A2H vs. ConH & 2,879 & A2S vs. ConS & 6,253 \\
\hline A3H vs. ConH & 3,544 & A3S vs. ConS & 8,978 \\
\hline A4H vs. ConH & 4,990 & A4S vs. ConS & 7,242 \\
\hline A2H vs. A1H & 2,788 & A2S vs. A1S & 1,553 \\
\hline A3H vs. A1H & 1,890 & A3S vs. A1S & 1,833 \\
\hline A4H vs. A1H & 1,222 & A4S vs. A1S & 1,102 \\
\hline A3H vs. A2H & 1,367 & A3S vs. A2S & 2,915 \\
\hline A4H vs. A2H & 2,229 & A4S vs. A2S & 1,394 \\
\hline A4H vs. A3H & 1,527 & A4S vs. A3S & 1,492 \\
\hline
\end{tabular}

Fig. 1. Age-related alterations of gene expression in the hippocampus and striatum after exposure of monkeys to heroin. Heroin was injected into monkeys of different ages (A1, A2, A3, and A4), and differentially expressed genes (DEGs) were analyzed using RNA sequencing technology. Genes that satisfied Ifold changel $\geq 2$ conditions in at least one of the total comparison pairs were selected. (A) Heat map analysis of DEGs among the hippocampus $(\mathrm{H})$ of control $(\mathrm{ConH})$ and heroin-treated $(\mathrm{A} 1 \mathrm{H}, \mathrm{A} 2 \mathrm{H}, \mathrm{A} 3 \mathrm{H}$, and $\mathrm{A} 4 \mathrm{H})$ animals. (B) Heat map analysis of DEGs among the striatum (S) of control (ConS) and heroin-treated (A1S, A2S, A3S, and A4S) animals. (C) Comparison of DEGs among groups.

A1, monkey aged 1 year; $A 2$, monkey aged $3-4$ years; $A 3$, monkey aged $6-8$ years; A4, monkey aged over 11 years. 
down-regulated in $\mathrm{A} 1 \mathrm{H}, \mathrm{A} 2 \mathrm{H}, \mathrm{A} 3 \mathrm{H}$, and $\mathrm{A} 4 \mathrm{H}$, respectively, compared to ConH (Fig. 1C). Among the herointreated groups, the number of DEGs was smallest between $\mathrm{A} 1 \mathrm{H}$ and $\mathrm{A} 4 \mathrm{H}(1,222$ transcripts), while the number of DEGs was largest between $\mathrm{A} 2 \mathrm{H}$ and $\mathrm{A} 4 \mathrm{H}(2,229$ transcripts). Of the 8,708 transcripts in the striatum, 7,382, $6,253,8,978$, and 7,242 transcripts were up-/down-regulated in A1S, A2S, A3S, and A4S, respectively, compared to ConS (Fig. 1C). When comparing genes differentially expressed in the striatum among the heroin-treated groups, the number between $\mathrm{A} 1 \mathrm{~S}$ and $\mathrm{A} 4 \mathrm{~S}$ was the smallest $(1,102$ transcripts), while that between $\mathrm{A} 3 \mathrm{~S}$ and $\mathrm{A} 2 \mathrm{~S}$ was the largest (2,915 transcripts), showing a pattern consistent with the hippocampus results.

Table 1. Gene ontology (GO) annotation of genes differentially expressed in the hippocampus of cynomolgus monkeys exposed to heroin relative to control (unexposed) animals

\begin{tabular}{|c|c|c|c|}
\hline Category & Term & Count & $p$ value \\
\hline \multicolumn{4}{|l|}{$\mathrm{A} 1 \mathrm{H}$ vs. ConH } \\
\hline GOTERM_BP_DIRECT & GO:0030036 - actin cytoskeleton organization & 10 & 0.007 \\
\hline GOTERM_BP_DIRECT & GO:0008380-RNA splicing & 11 & 0.019 \\
\hline GOTERM_BP_DIRECT & GO:0015031 - protein transport & 16 & 0.024 \\
\hline GOTERM BP DIRECT & GO:0006357 - regulation of transcription from RNA polymerase II promoter & 10 & 0.037 \\
\hline GOTERM_BP_DIRECT & GO:0007156 - homophilic cell adhesion via plasma membrane adhesion molecules & 6 & 0.046 \\
\hline KEGG_PATHWAY & mcf04010: MAPK signaling pathway & 78 & $<0.001$ \\
\hline KEGG_PATHWAY & mcf00970:Aminoacyl-tRNA biosynthesis & 21 & $<0.001$ \\
\hline KEGG_PATHWAY & mcf01130:Biosynthesis of antibiotics & 72 & $<0.001$ \\
\hline KEGG_PATHWAY & mcf04141:Protein processing in endoplasmic reticulum & 57 & $<0.001$ \\
\hline KEGG_PATHWAY & mcf01100:Metabolic pathways & 313 & $<0.001$ \\
\hline \multicolumn{4}{|l|}{$\mathrm{A} 2 \mathrm{H}$ vs. $\mathrm{ConH}$} \\
\hline GOTERM_BP_DIRECT & GO:0030036 - actin cytoskeleton organization & 7 & 0.018 \\
\hline GOTERM_BP_DIRECT & GO:0016192 - vesicle-mediated transport & 11 & 0.020 \\
\hline GOTERM_BP_DIRECT & GO:0051260 - protein homooligomerization & 8 & 0.035 \\
\hline KEGG_PATHWAY & mcf04723:Retrograde endocannabinoid signaling & 28 & $<0.001$ \\
\hline KEGG_PATHWAY & mcf04727:GABAergic synapse & 22 & $<0.001$ \\
\hline KEGG_PATHWAY & mcf05033:Nicotine addiction & 14 & $<0.001$ \\
\hline KEGG_PATHWAY & mcf04720:Long-term potentiation & 19 & $<0.001$ \\
\hline KEGG_PATHWAY & mcf04724:Glutamatergic synapse & 26 & $<0.001$ \\
\hline \multicolumn{4}{|l|}{$\mathrm{A} 3 \mathrm{H}$ vs. $\mathrm{ConH}$} \\
\hline GOTERM_BP_DIRECT & GO:0030036 - actin cytoskeleton organization & 9 & 0.002 \\
\hline GOTERM_BP_DIRECT & GO:0006357 - regulation of transcription from RNA polymerase II promoter & 10 & 0.003 \\
\hline GOTERM_BP_DIRECT & GO:0051260 - protein homooligomerization & 9 & 0.029 \\
\hline KEGG_PATHWAY & mcf04727:GABAergic synapse & 27 & $<0.001$ \\
\hline KEGG_PATHWAY & mcf04723:Retrograde endocannabinoid signaling & 30 & $<0.001$ \\
\hline KEGG_PATHWAY & mcf04724:Glutamatergic synapse & 31 & $<0.001$ \\
\hline KEGG_PATHWAY & mcf05033:Nicotine addiction & 16 & $<0.001$ \\
\hline KEGG_PATHWAY & mcf04720:Long-term potentiation & 22 & $<0.001$ \\
\hline \multicolumn{4}{|l|}{$\mathrm{A} 4 \mathrm{H}$ vs. $\mathrm{ConH}$} \\
\hline GOTERM_BP_DIRECT & GO:0008380-RNA splicing & 11 & 0.009 \\
\hline GOTERM_BP_DIRECT & GO:0015031 - protein transport & 16 & 0.010 \\
\hline GOTERM_BP_DIRECT & GO:0046907 - intracellular transport & 4 & 0.038 \\
\hline GOTERM_BP_DIRECT & GO:0006397-mRNA processing & 12 & 0.043 \\
\hline GOTERM_BP_DIRECT & GO:0030036 - actin cytoskeleton organization & 8 & 0.047 \\
\hline KEGG_PATHWAY & mcf04727:GABAergic synapse & 31 & $<0.001$ \\
\hline KEGG_PATHWAY & mcf04010:MAPK signaling pathway & 72 & $<0.001$ \\
\hline KEGG_PATHWAY & mcf04723:Retrograde endocannabinoid signaling & 33 & $<0.001$ \\
\hline KEGG_PATHWAY & mcf04141:Protein processing in endoplasmic reticulum & 50 & 0.001 \\
\hline KEGG_PATHWAY & mcf01130:Biosynthesis of antibiotics & 62 & 0.002 \\
\hline
\end{tabular}

$\mathrm{A} 1 \mathrm{H}$, hippocampus of the monkey aged 1 year; $\mathrm{A} 2 \mathrm{H}$, hippocampus of the monkey aged $3-4$ years; $\mathrm{A} 3 \mathrm{H}$, hippocampus of the monkey aged $6-8$ years; $\mathrm{A} 4 \mathrm{H}$, hippocampus of the monkey aged over 11 years; $\mathrm{ConH}$, hippocampus of control; MAPK, mitogen-activated protein kinase; tRNA, transfer RNA; GABA, $\gamma$-aminobutyric acid; mRNA, messenger RNA. 
Table 2. Gene ontology (GO) annotation of genes differentially expressed in the hippocampus of cynomolgus monkeys of different ages exposed to heroin

\begin{tabular}{|c|c|c|c|}
\hline Category & Term & Count & $p$ value \\
\hline \multicolumn{4}{|l|}{$\mathrm{A} 2 \mathrm{H}$ vs. $\mathrm{A} 1 \mathrm{H}$} \\
\hline GOTERM_BP_DIRECT & GO:0030036 - actin cytoskeleton organization & 9 & $<0.001$ \\
\hline GOTERM_BP_DIRECT & GO:0007399 - nervous system development & 10 & 0.001 \\
\hline GOTERM_BP_DIRECT & GO:0007156-homophilic cell adhesion via plasma membrane adhesion molecules & 5 & 0.030 \\
\hline GOTERM_BP_DIRECT & GO:0015031 - protein transport & 11 & 0.032 \\
\hline GOTERM_BP_DIRECT & GO:0007218-neuropeptide signaling pathway & 4 & 0.043 \\
\hline KEGG_PATHWAY & mcf01130:Biosynthesis of antibiotics & 46 & $<0.001$ \\
\hline KEGG_PATHWAY & mcf01100:Metabolic pathways & 187 & $<0.001$ \\
\hline KEGG_PATHWAY & mcf00970:Aminoacyl-tRNA biosynthesis & 14 & $<0.001$ \\
\hline KEGG_PATHWAY & mcf04144:Endocytosis & 47 & 0.002 \\
\hline KEGG_PATHWAY & mcf04010:MAPK signaling pathway & 45 & 0.003 \\
\hline \multicolumn{4}{|l|}{$\mathrm{A} 3 \mathrm{H}$ vs. $\mathrm{A} 1 \mathrm{H}$} \\
\hline GOTERM_BP_DIRECT & GO:0030036 - actin cytoskeleton organization & 9 & $<0.001$ \\
\hline GOTERM_BP_DIRECT & GO:0046907-intracellular transport & 3 & 0.046 \\
\hline KEGG_PATHWAY & mcf04144:Endocytosis & 39 & $<0.001$ \\
\hline KEGG_PATHWAY & mcf04010:MAPK signaling pathway & 34 & 0.004 \\
\hline KEGG_PATHWAY & mcf00310:Lysine degradation & 11 & 0.008 \\
\hline KEGG_PATHWAY & mcf04261:Adrenergic signaling in cardiomyocytes & 22 & 0.008 \\
\hline KEGG_PATHWAY & mcf00511:Other glycan degradation & 6 & 0.014 \\
\hline \multicolumn{4}{|l|}{$\mathrm{A} 4 \mathrm{H}$ vs. $\mathrm{A} 1 \mathrm{H}$} \\
\hline GOTERM_BP_DIRECT & GO:0006511 - ubiquitin-dependent protein catabolic process & 6 & 0.024 \\
\hline GOTERM_BP_DIRECT & GO:0000166 - nucleotide binding & 12 & 0.017 \\
\hline KEGG_PATHWAY & mcf00970:Aminoacyl-tRNA biosynthesis & 8 & 0.005 \\
\hline KEGG_PATHWAY & mcf04024:cAMP signaling pathway & 19 & 0.009 \\
\hline KEGG_PATHWAY & mcf05010:Alzheimer's disease & 19 & 0.018 \\
\hline KEGG_PATHWAY & mcf04144:Endocytosis & 21 & 0.033 \\
\hline KEGG_PATHWAY & mcf04720:Long-term potentiation & 8 & 0.042 \\
\hline \multicolumn{4}{|l|}{$\mathrm{A} 3 \mathrm{H}$ vs. $\mathrm{A} 2 \mathrm{H}$} \\
\hline KEGG_PATHWAY & mcf04010:MAPK signaling pathway & 29 & 0.002 \\
\hline KEGG_PATHWAY & mcf04144:Endocytosis & 29 & 0.004 \\
\hline KEGG_PATHWAY & mcf04666:Fc gamma R-mediated phagocytosis & 12 & 0.024 \\
\hline KEGG_PATHWAY & mcf00511:Other glycan degradation & 5 & 0.026 \\
\hline KEGG_PATHWAY & mcf05204:Chemical carcinogenesis & 10 & 0.026 \\
\hline \multicolumn{4}{|l|}{$\mathrm{A} 4 \mathrm{H}$ vs. $\mathrm{A} 2 \mathrm{H}$} \\
\hline GOTERM_BP_DIRECT & GO:0030036 - actin cytoskeleton organization & 9 & $<0.001$ \\
\hline GOTERM_BP_DIRECT & GO:0007399 - nervous system development & 7 & 0.036 \\
\hline GOTERM_BP_DIRECT & GO:0006897-endocytosis & 5 & 0.036 \\
\hline KEGG_PATHWAY & mcf00970:Aminoacyl-tRNA biosynthesis & 14 & $<0.001$ \\
\hline KEGG_PATHWAY & mcf04010:MAPK signaling pathway & 42 & $<0.001$ \\
\hline KEGG_PATHWAY & mcf01130:Biosynthesis of antibiotics & 39 & $<0.001$ \\
\hline KEGG_PATHWAY & mcf01200:Carbon metabolism & 24 & 0.002 \\
\hline KEGG_PATHWAY & mcf04066:HIF-1 signaling pathway & 21 & 0.002 \\
\hline \multicolumn{4}{|l|}{$\mathrm{A} 4 \mathrm{H}$ vs. $\mathrm{A} 3 \mathrm{H}$} \\
\hline GOTERM_BP_DIRECT & GO:0030036 - actin cytoskeleton organization & 6 & 0.007 \\
\hline GOTERM_BP_DIRECT & GO:0015031 - protein transport & 9 & 0.009 \\
\hline GOTERM_BP_DIRECT & GO:0006412- translation & 16 & 0.024 \\
\hline GOTERM_BP_DIRECT & GO:0046907-intracellular transport & 3 & 0.037 \\
\hline KEGG_PATHWAY & mcf00310:Lysine degradation & 9 & 0.025 \\
\hline KEGG_PATHWAY & mcf00970:Aminoacyl-tRNA biosynthesis & 8 & 0.027 \\
\hline KEGG_PATHWAY & mcf00760:Nicotinate and nicotinamide metabolism & 6 & 0.043 \\
\hline
\end{tabular}

$\mathrm{A} 1 \mathrm{H}$, hippocampus of the monkey aged 1 year; $\mathrm{A} 2 \mathrm{H}$, hippocampus of the monkey aged $3-4$ years; $\mathrm{A} 3 \mathrm{H}$, hippocampus of the monkey aged $6-8$ years; $\mathrm{A} 4 \mathrm{H}$, hippocampus of the monkey aged over 11 years; tRNA, transfer RNA; MAPK, mitogen-activated protein kinase; cAMP, cyclic adenosine monophosphate; HIF-1, hypoxia-inducible factor 1. 


\section{Gene Enrichment and Functional Annotation}

We analyzed the GO annotation and KEGG pathways of genes differentially expressed among control and heroin-treated groups. In the BP category, we selected the top five networks (including the KEGG pathway). As pre- sented in Table 1, genes associated with actin cytoskeleton organization (GO:0030036) were differentially expressed in the hippocampus of all heroin-treated groups $(\mathrm{A} 1 \mathrm{H}, \mathrm{A} 2 \mathrm{H}, \mathrm{A} 3 \mathrm{H}$, and $\mathrm{A} 4 \mathrm{H})$ compared to $\mathrm{ConH}$. On the basis of our KEGG pathway analysis, genes related to ret-

Table 3. Gene ontology (GO) annotation of genes differentially expressed in the striatum of cynomolgus monkeys exposed to heroin relative to control (unexposed) monkeys

\begin{tabular}{|c|c|c|c|}
\hline Category & Term & Count & $p$ value \\
\hline \multicolumn{4}{|l|}{ A1S vs. ConS } \\
\hline GOTERM_BP_DIRECT & GO:0006897 - endocytosis & 10 & $<0.001$ \\
\hline GOTERM_BP_DIRECT & GO:0008380-RNA splicing & 14 & 0.003 \\
\hline GOTERM_BP_DIRECT & GO:0007264-small GTPase mediated signal transduction & 37 & 0.019 \\
\hline GOTERM_BP_DIRECT & GO:0015031 - protein transport & 19 & 0.020 \\
\hline GOTERM_BP_DIRECT & GO:0030036 - actin cytoskeleton organization & 10 & 0.033 \\
\hline KEGG_PATHWAY & mcf04720:Long-term potentiation & 34 & $<0.001$ \\
\hline KEGG_PATHWAY & mcf04722:Neurotrophin signaling pathway & 54 & $<0.001$ \\
\hline KEGG_PATHWAY & mcf04141:Protein processing in endoplasmic reticulum & 71 & $<0.001$ \\
\hline KEGG_PATHWAY & mcf04713:Circadian entrainment & 43 & $<0.001$ \\
\hline KEGG_PATHWAY & mcf04921:Oxytocin signaling pathway & 66 & $<0.001$ \\
\hline \multicolumn{4}{|l|}{ A2S vs. ConS } \\
\hline GOTERM_BP_DIRECT & GO:0015031 - protein transport & 20 & 0.001 \\
\hline GOTERM_BP_DIRECT & GO:0008380-RNA splicing & 13 & 0.004 \\
\hline GOTERM_BP_DIRECT & GO:0006810 - transport & 18 & 0.024 \\
\hline GOTERM_BP_DIRECT & GO:0006412-translation & 38 & 0.048 \\
\hline GOTERM_BP_DIRECT & GO:0030036 - actin cytoskeleton organization & 9 & 0.049 \\
\hline KEGG_PATHWAY & mcf01200:Carbon metabolism & 52 & $<0.001$ \\
\hline KEGG_PATHWAY & mcf04141:Protein processing in endoplasmic reticulum & 67 & $<0.001$ \\
\hline KEGG_PATHWAY & mcf01130:Biosynthesis of antibiotics & 84 & $<0.001$ \\
\hline KEGG_PATHWAY & mcf04921:Oxytocin signaling pathway & 62 & $<0.001$ \\
\hline KEGG_PATHWAY & mcf04720:Long-term potentiation & 30 & $<0.001$ \\
\hline \multicolumn{4}{|l|}{ A3S vs. ConS } \\
\hline GOTERM_BP_DIRECT & GO:0006897 - endocytosis & 9 & 0.018 \\
\hline GOTERM_BP_DIRECT & GO:0007399- nervous system development & 14 & 0.018 \\
\hline GOTERM_BP_DIRECT & GO:0006914-autophagy & 12 & 0.019 \\
\hline GOTERM_BP_DIRECT & GO:0015031 - protein transport & 21 & 0.026 \\
\hline GOTERM_BP_DIRECT & GO:0030036 - actin cytoskeleton organization & 11 & 0.035 \\
\hline KEGG_PATHWAY & mcf04261:Adrenergic signaling in cardiomyocytes & 73 & $<0.001$ \\
\hline KEGG_PATHWAY & mcf04720:Long-term potentiation & 38 & $<0.001$ \\
\hline KEGG_PATHWAY & mcf04728:Dopaminergic synapse & 62 & $<0.001$ \\
\hline KEGG_PATHWAY & mcf04722:Neurotrophin signaling pathway & 61 & $<0.001$ \\
\hline KEGG_PATHWAY & mcf04015:Rap1 signaling pathway & 98 & $<0.001$ \\
\hline \multicolumn{4}{|l|}{ A4S vs. ConS } \\
\hline GOTERM_BP_DIRECT & GO:0008380-RNA splicing & 14 & 0.003 \\
\hline GOTERM_BP_DIRECT & GO:0006897 - endocytosis & 9 & 0.005 \\
\hline GOTERM_BP_DIRECT & GO:0015031 - protein transport & 19 & 0.016 \\
\hline GOTERM_BP_DIRECT & GO:0006397-mRNA processing & 15 & 0.036 \\
\hline GOTERM_BP_DIRECT & GO:0006914-autophagy & 10 & 0.047 \\
\hline KEGG_PATHWAY & mcf04720:Long-term potentiation & 33 & $<0.001$ \\
\hline KEGG_PATHWAY & mcf01200:Carbon metabolism & 54 & $<0.001$ \\
\hline KEGG_PATHWAY & mcf01130:Biosynthesis of antibiotics & 88 & $<0.001$ \\
\hline KEGG_PATHWAY & mcf05010:Alzheimer’s disease & 82 & $<0.001$ \\
\hline KEGG_PATHWAY & mcf04261:Adrenergic signaling in cardiomyocytes & 62 & $<0.001$ \\
\hline
\end{tabular}

$\mathrm{A} 1 \mathrm{~S}$, striatum of the monkey aged 1 year; $\mathrm{A} 2 \mathrm{~S}$, striatum of the monkey aged $3-4$ years; A3S, striatum of the monkey aged $6-8$ years; A4S, striatum of the monkey aged over 11 years; ConS, striatum of control; GTPase, guanosine triphosphatase; mRNA, messenger RNA. 
rograde endocannabinoid signaling (mcf04723) and GABAergic synapse (mcf04727) were differentially expressed in the $\mathrm{A} 2 \mathrm{H}, \mathrm{A} 3 \mathrm{H}$, and $\mathrm{A} 4 \mathrm{H}$ groups compared to $\mathrm{ConH}$. On the other hand, when we analyzed the GO annotation and KEGG pathways of genes differentially expressed among $\mathrm{A} 1 \mathrm{H}, \mathrm{A} 2 \mathrm{H}, \mathrm{A} 3 \mathrm{H}$, and $\mathrm{A} 4 \mathrm{H}$ groups, genes related to nervous system development (GO:0007399) and neuropeptide signaling pathways (GO:0007218) were differentially expressed in $\mathrm{A} 2 \mathrm{H}$ compared to $\mathrm{A} 1 \mathrm{H}$ (Table 2). In addition, we found that 19 genes (mcf05010) related to Alzheimer's disease (AD) were differentially expressed between $\mathrm{A} 1 \mathrm{H}$ and $\mathrm{A} 4 \mathrm{H}$.

In a GO analysis of the striatum, genes associated with endocytosis (GO:0006897) were differentially expressed

Table 4. Gene ontology (GO) annotation of genes differentially expressed in the striatum of cynomolgus monkeys of different ages exposed to heroin

\begin{tabular}{|c|c|c|c|}
\hline Category & Term & Count & $p$ value \\
\hline \multicolumn{4}{|l|}{ A2S vs. A1S } \\
\hline KEGG_PATHWAY & mcf04144:Endocytosis & 29 & 0.007 \\
\hline KEGG_PATHWAY & mcf04713:Circadian entrainment & 14 & 0.007 \\
\hline KEGG_PATHWAY & mcf00330:Arginine and proline metabolism & 9 & 0.017 \\
\hline KEGG_PATHWAY & mcf01130:Biosynthesis of antibiotics & 25 & 0.017 \\
\hline KEGG_PATHWAY & mcf04530:Tight junction & 17 & 0.021 \\
\hline \multicolumn{4}{|l|}{ A3S vs. A1S } \\
\hline GOTERM_BP_DIRECT & GO:0006897-endocytosis & 6 & 0.003 \\
\hline GOTERM_BP_DIRECT & GO:0030036 - actin cytoskeleton organization & 7 & 0.003 \\
\hline KEGG_PATHW'- & mcf04071:Sphingolipid signaling pathway & 22 & $<0.001$ \\
\hline KEGG_PATHWAY & mcf04728:Dopaminergic synapse & 20 & 0.004 \\
\hline KEGG_PATHWAY & mcf05142:Chagas disease (American trypanosomiasis) & 17 & 0.005 \\
\hline KEGG_PATHWAY & mcf00230:Purine metabolism & 26 & 0.005 \\
\hline KEGG_PATHWAY & mcf05203:Viral carcinogenesis & 29 & 0.006 \\
\hline \multicolumn{4}{|l|}{ A4S vs. A1S } \\
\hline KEGG_PATHWAY & mcf05203:Viral carcinogenesis & 21 & 0.002 \\
\hline KEGG_PATHWAY & mcf04612:Antigen processing and presentation & 9 & 0.019 \\
\hline KEGG_PATHWAY & mcf04144:Endocytosis & 21 & 0.024 \\
\hline KEGG_PATHWAY & mcf00330:Arginine and proline metabolism & 7 & 0.033 \\
\hline KEGG_PATHWAY & mcf00380:Tryptophan metabolism & 6 & 0.044 \\
\hline \multicolumn{4}{|l|}{ A3S vs. A2S } \\
\hline GOTERM_BP_DIRECT & GO:0030036 - actin cytoskeleton organization & 7 & 0.026 \\
\hline KEGG_PATHWAY & mcf04142:Lysosome & 31 & $<0.001$ \\
\hline KEGG_PATHWAY & mcf01100:Metabolic pathways & 196 & $<0.001$ \\
\hline KEGG_PATHWAY & mcf04144:Endocytosis & 49 & 0.002 \\
\hline KEGG_PATHWAY & mcf01130:Biosynthesis of antibiotics & 44 & 0.003 \\
\hline KEGG_PATHWAY & mcf01230:Biosynthesis of amino acids & 20 & 0.004 \\
\hline \multicolumn{4}{|l|}{ A4S vs. A2S } \\
\hline GOTERM_BP_DIRECT & GO:0006914-autophagy & 6 & 0.006 \\
\hline GOTERM_BP_DIRECT & GO:0055114-oxidation-reduction process & 5 & 0.038 \\
\hline KEGG_PATHWAY & mcf05203:Viral carcinogenesis & 25 & 0.002 \\
\hline KEGG_PATHWAY & mcf05010:Alzheimer’s disease & 25 & 0.002 \\
\hline KEGG_PATHWAY & mcf04961:Endocrine and other factor-regulated calcium reabsorption & 9 & 0.004 \\
\hline KEGG_PATHWAY & mcf04720:Long-term potentiation & 11 & 0.005 \\
\hline KEGG_PATHWAY & mcf04728:Dopaminergic synapse & 15 & 0.017 \\
\hline \multicolumn{4}{|l|}{ A4S vs. A3S } \\
\hline GOTERM_BP_DIRECT & GO:0006914-autophagy & 7 & 0.001 \\
\hline KEGG_PATHWAY & mcf00480:Glutathione metabolism & 10 & 0.006 \\
\hline KEGG_PATHWAY & mcf01100:Metabolic pathways & 106 & 0.008 \\
\hline KEGG_PATHWAY & mcf01230:Biosynthesis of amino acids & 12 & 0.016 \\
\hline KEGG_PATHWAY & mcf01130:Biosynthesis of antibiotics & 25 & 0.016 \\
\hline KEGG_PATHWAY & mcf00563:Glycosylphosphatidylinositol-anchor biosynthesis & 6 & 0.019 \\
\hline
\end{tabular}

A1S, striatum of the monkey aged 1 year; A2S, striatum of the monkey aged $3-4$ years; A3S, striatum of the monkey aged $6-8$ years; A4S, striatum of the monkey aged over 11 years. 
in A1S, A3S, and A4S compared to ConS (Table 3). Furthermore, genes associated with RNA splicing (GO:0008380) were differentially expressed in A1S, A2S, and A4S compared to ConS. Autophagy-related genes (GO:0006914) were differentially expressed in A3S and A4S compared to ConS. In our KEGG pathway analysis of the striatum, genes related to long-term potentiation (LTP) (mcf04720) were differentially expressed in A1S, A2S, A3S, and A4S compared to ConS (Table 3). On the other hand, when we analyzed the GO annotation and KEGG pathways of genes differentially expressed among A1S, $\mathrm{A} 2 \mathrm{~S}, \mathrm{~A} 3 \mathrm{~S}$, and $\mathrm{A} 4 \mathrm{~S}$, circadian entrainment-related genes (mcf04713) were differentially expressed in A2S vs. A1S in response to heroin (Table 4). Besides, we found that 25 genes (mcf05010) related to AD were differentially expressed between $\mathrm{A} 2 \mathrm{~S}$ and $\mathrm{A} 4 \mathrm{~S}$.

\section{Validation of DEGs}

Of genes differentially expressed in the hippocampus, genes related to the MAPK signaling pathway (STMN1, FGF14, and MAPT), GABAergic synapses (GABBR2 and $G A D 1)$, and retrograde endocannabinoid signaling (GRIA2) were validated using RT-qPCR. STMN1 was significantly downregulated in the $\mathrm{A} 2 \mathrm{H}$ group compared to the control group, while it was significantly upregulated in the $\mathrm{A} 1 \mathrm{H}$ group compared to the control group (Supplementary Table 2 and Fig. 2). FGF14 and GABBR2 were significantly decreased in three heroin-treated $(\mathrm{A} 1 \mathrm{H}, \mathrm{A} 3 \mathrm{H}$, and $\mathrm{A} 4 \mathrm{H})$ compared to the control group, while MAPT, GAD1, and GRIA2 were significantly downregulated in all heroin-treated groups. However, expression level of GABBR2 also decreased in all heroin-treated groups compared to the control group, showing a significant decrease in $\mathrm{A} 1 \mathrm{H}$, $\mathrm{A} 3 \mathrm{H}$, and $\mathrm{A} 4 \mathrm{H}$ (Supplementary Table 2 and Fig. 2).

Of genes differentially expressed in the striatum, genes related to the neurotrophin signaling pathway (NTRK1 and $N G F R)$, oxytocin signaling pathway (CACNG3 and NFATC4), autophagy (ATG5), and dopaminergic syn-
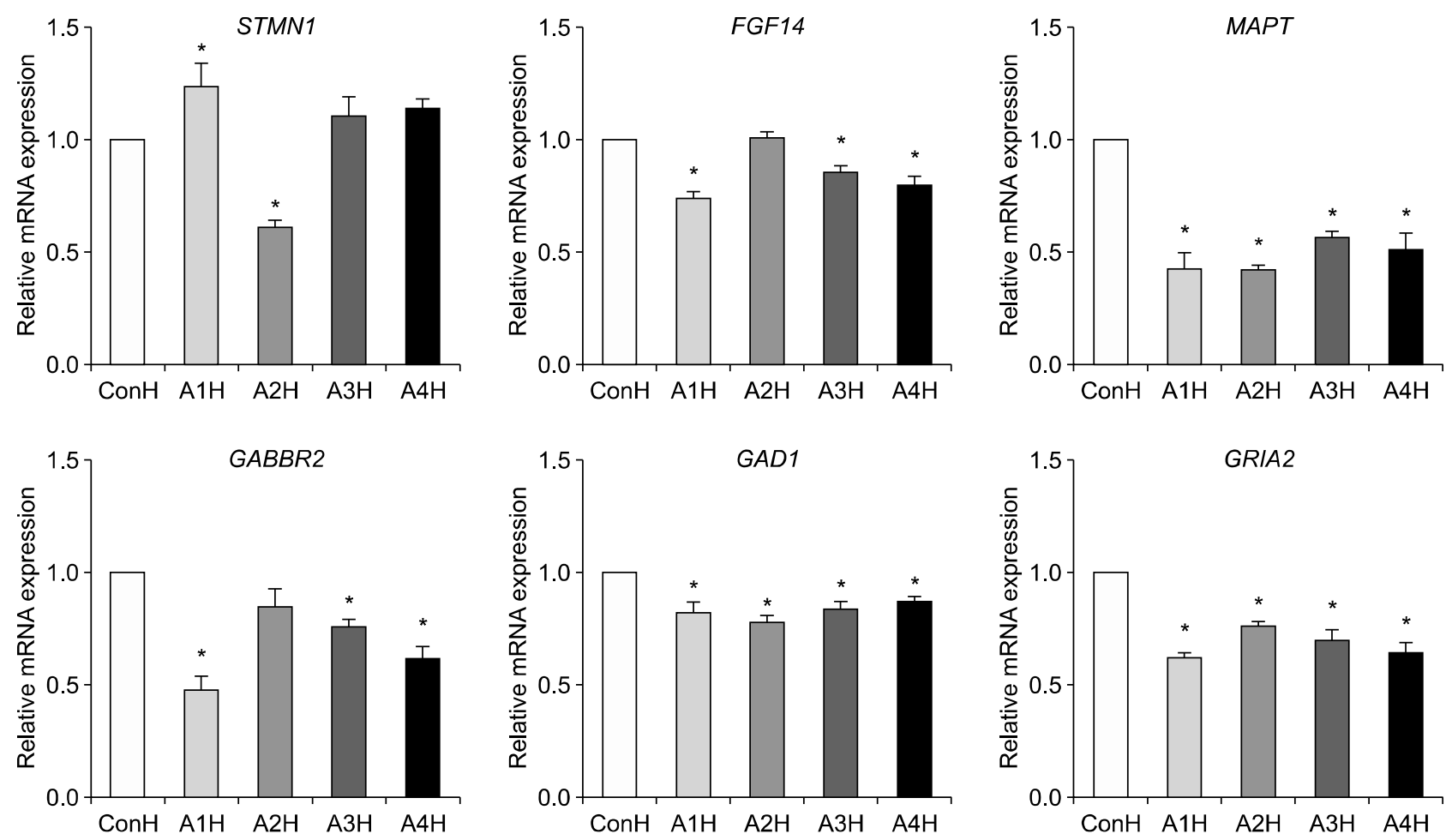

Fig. 2. Validation of differentially-expressed genes identified in the hippocampus by RNA sequencing. After a single injection of heroin in monkeys of different ages, differentially expressed genes in the hippocampus $(\mathrm{H})$ were analyzed using $\mathrm{RT}$-qPCR. Two monkeys per group $(\mathrm{ConH}$, $\mathrm{A} 1 \mathrm{H}$, $\mathrm{A} 2 \mathrm{H}$, $\mathrm{A} 3 \mathrm{H}$, and $\mathrm{A} 4 \mathrm{H})$ were used for the experiment. Genes related to MAPK signaling (STMN1, FGF14, and MAPT), GABAergic synapses (GABBR2 and $G A D 1)$, and retrograde endocannabinoid signaling $(G R I A 2)$ were analyzed. Data are represented as the mean \pm standard error of the mean $(\mathrm{n}=3)$. Data were analyzed using one-way ANOVA followed by Tukey's honestly significant difference post-hoc test.

mRNA, messenger RNA; RT-qPCR, real-time quantitative PCR; MAPK, mitogen-activated protein kinase; GABA, $\gamma$-aminobutyric acid.

*Significantly different from the control group $(p<0.05)$. 

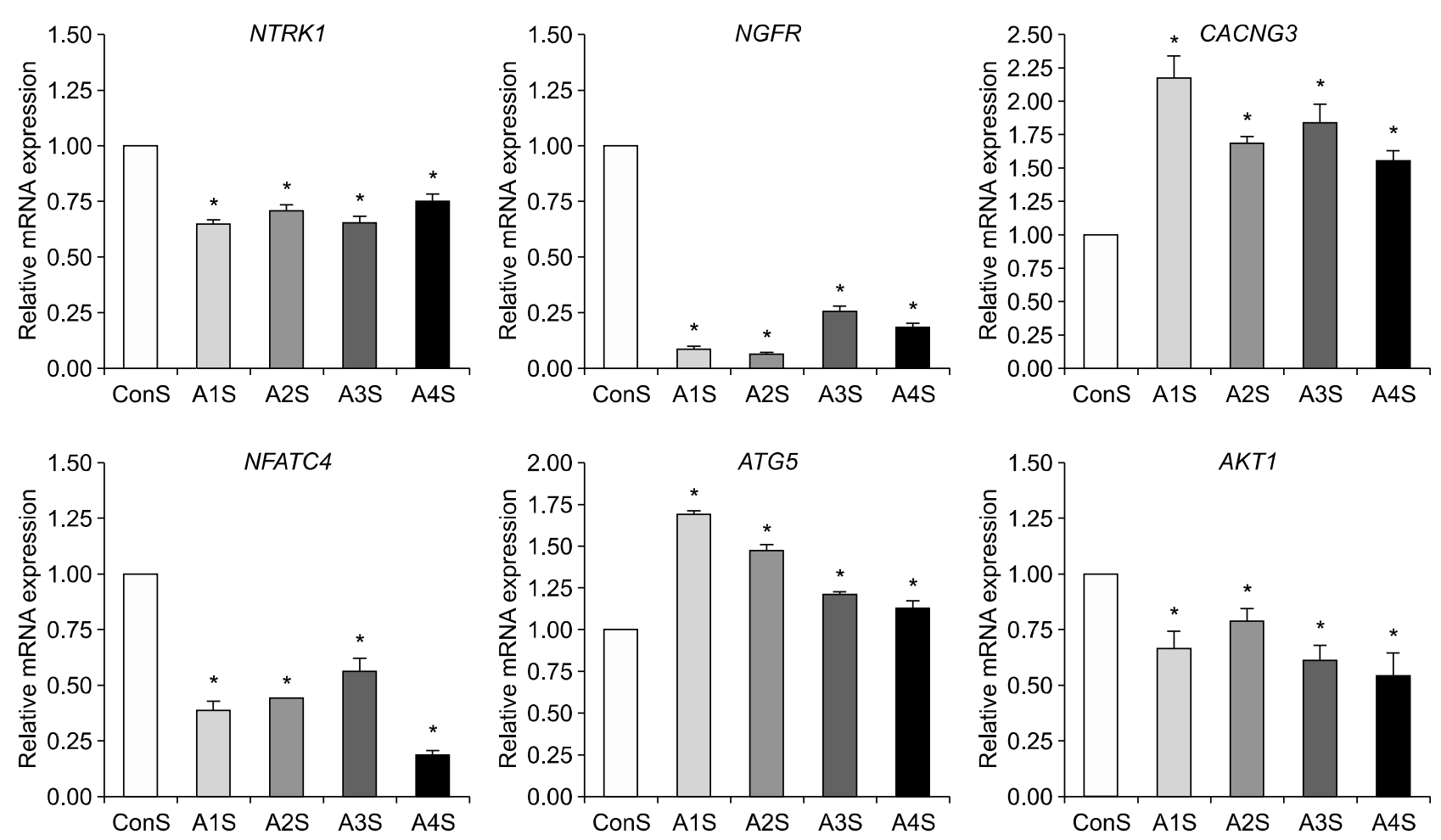

Fig. 3. Validation of differentially-expressed genes identified in the striatum by RNA sequencing. After a single injection of heroin to monkeys of different ages, differentially expressed genes in the striatum (S) were analyzed using RT-qPCR. Two monkeys per group (ConS, A1S, A2S, A3S, and A4S) were used for the experiment. Genes related to neurotrophin signaling (NTRK1 and NGFR), oxytocin signaling (CACNG3 and NFATC4), autophagy (ATC5), and the dopaminergic synapse ( $A K T 1)$ were analyzed. Data are represented as the mean \pm standard error of the mean $(\mathrm{n}=3)$. Data were analyzed using one-way ANOVA followed by Tukey's honestly significant difference post-hoc test.

mRNA, messenger RNA; RT-qPCR, real-time quantitative PCR.

*Significantly different from the control group $(p<0.05)$.

apses $(A K T 1)$ were validated using RT-qPCR. Expression levels of NTRK1 and NGFR were significantly downregulated in heroin-treated groups (A1S, A2S, A3S, and A4S) compared to the control (ConS) by both RNA-Seq (Supplementary Table 3) and RT-qPCR (Fig. 3). On the other hand, the expression level of CACNG3 was increased in all heroin-treated groups compared to the control, while that of NFATC4 was decreased in all heroin-treated groups compared to the control by both RNA-Seq (Supplementary Table 3) and RT-qPCR (Fig. 3). ATG 5 was increased in all heroin-treated groups compared to the control group, showing a tendency to decrease with age in heroin-treated groups. Conversely, AKT1 was significantly decreased in all heroin-treated groups compared to the control (Fig. 3). Taken together, expression patterns of all the validated genes in RT-qPCR were the same as the result obtained from RNA-Seq.

\section{DISCUSSION}

The short-term effects of heroin include dry mouth, skin flushing, vomiting, nausea, and severe itching. In addition, heroin induces slowness of heart function and breathing as well as somnolence. These changes can cause coma and permanent brain damage. Despite these detrimental effects, people of all ages are illegally using heroin to induce euphoria. To identify the effects of heroin on the brain at the molecular and pathophysiological levels depending on age, we investigated global alterations in gene expression and functional networks in the hippocampus and striatum of cynomolgus monkeys, which have a brain structure and function very similar to humans.

In the present study, we found that gene expression of $\mathrm{A} 1 \mathrm{H}$ and $\mathrm{A} 4 \mathrm{H}$ was similar, as was that of $\mathrm{A} 2 \mathrm{H}$ and $\mathrm{A} 3 \mathrm{H}$. These findings are consistent with our previous study, in which cynomolgus monkeys exposed to the psychostimu- 
lant methamphetamine showed similar gene expression patterns according to age [20]. These findings imply that age affects the way in which addictive drugs alter gene expression, regardless of the type of drug (opiate or psychostimulant). Based on our results that expression patterns of genes in $\mathrm{A} 1 \mathrm{H}$ were closer to those of $\mathrm{A} 4 \mathrm{H}$ than in other animals, it is supposed that hippocampi of animals at these ages show a similar response to heroin. The ages of animals in $\mathrm{A} 1 \mathrm{H}$ and $\mathrm{A} 4 \mathrm{H}$ are equivalent to the ages of children and middle-aged or older humans, respectively. Human children are especially vulnerable to drugs [25]. Therefore, it is believed that the hippocampus of $\mathrm{A} 1 \mathrm{H}$ and $\mathrm{A} 4 \mathrm{H}$ animals is vulnerable to heroin, exposure to which causes similar gene expression in $\mathrm{A} 1 \mathrm{H}$ and $\mathrm{A} 4 \mathrm{H}$ at a molecular level. The ages of animals in groups $\mathrm{A} 2 \mathrm{H}$ and $\mathrm{A} 3 \mathrm{H}$ are comparable to human adolescence, when brain and body systems experience crucial maturational rearrangements [26]. Among the heroin-treated groups, the number of DEGs between $\mathrm{A} 2 \mathrm{H}$ and $\mathrm{A} 3 \mathrm{H}$ was somewhat smaller than that among other age groups. This indicates that the hippocampus tissues of adolescents and young adults exhibit common molecular changes on exposure to heroin. Considering that the use of addictive drugs (including heroin) is associated with dopaminergic activation in the striatum [27], the alteration of gene expression by activated dopaminergic synapses may occur. Unlike the hippocampus, the number of DEGs between A2S and A3S in the striatum was the largest among the heroin-treated groups. Because animals in the $\mathrm{A} 2 \mathrm{~S}$ and $\mathrm{A} 3 \mathrm{~S}$ age groups experience critical maturational rearrangements, it is likely that even a single exposure to heroin induces molecular changes in the striatum.

In the present study, heroin treatment led to up- or downregulation of genes related to actin cytoskeleton organization in the hippocampus of all heroin-treated groups. The actin cytoskeleton is responsible for regulating junctional integrity and cellular remodeling under pathological and physiological states [28]. Therefore, the heroin-induced reorganization of the actin cytoskeleton can affect a variety of cellular functions in the brain, such as motility, cellular signaling, cell adhesion, and intracellular trafficking. On the other hand, $\mathrm{A} 2 \mathrm{H}, \mathrm{A} 3 \mathrm{H}$, and $\mathrm{A} 4 \mathrm{H}$ groups exhibited up- or downregulation of genes related to retrograde endocannabinoid signaling (mcf04723) compared to ConH. Excitatory and inhibitory transmission in the brain leads to feedback inhibition through the activation of presynaptic CB1 receptors by endocannabinoids (eCBs) [29]. A previous report has shown that acute exposure to cocaine and alcohol results in the disruption of eCB-mediated synaptic plasticity in animal models [30]. Our findings suggest that heroin, an addictive drug, may also induce an imbalanced eCB-mediated synaptic plasticity, promoting drug-seeking behaviors and the stress response. Taken together, these results suggest that it is likely that even a single exposure of heroin disrupts synaptic plasticity.

In this study, genes associated with nervous system development were differentially expressed between $\mathrm{A} 1 \mathrm{H}$ and $\mathrm{A} 2 \mathrm{H}$ groups. Monkeys in groups $\mathrm{A} 1$ and $\mathrm{A} 2$ are similar in age to humans in their early childhood and teenage years. These are ages in which structural brain development and neurophysiological mechanisms (such as increase of myelination and axon density) actively occur [31]. Therefore, exposure to heroin at these ages can cause alterations in genes related to nervous system development. On the other hand, we found that 19 genes associated with $A D$ were differentially expressed between $\mathrm{A} 1 \mathrm{H}$ and $\mathrm{A} 4 \mathrm{H}$. Animals in group $\mathrm{A} 4$ are equivalent to humans in their mid-thirties, whose brains have already stopped growing. Even a single exposure to heroin may lead to alterations in genes related to cognitive function in the hippocampus, possibly affecting the early onset of diseases like dementia.

In the present study, interestingly, autophagy-related genes (GO:0006914) were up- or downregulated in A3S and A4S compared to ConS. Autophagy is provoked by hypoxia, metabolic stress, and starvation and is responsible for removing intracellular aggregates and cytoplasmic organelles and promoting cellular senescence [32]. Previous researchers have demonstrated that chronic exposure to opiates (including heroin) promotes autophagy in rats [33] and in human addicts ranging in age from 19 to 40 years [34]. The present study also found that a single exposure to heroin induced the alteration of autophagy-related genes in the striatum tissues of monkeys of specific ages. Some research has demonstrated that autophagy-related genes are increased in brain-derived cells (including SH-SY5Y and C6 cells) and in the hippocampus tissues of rats in response to morphine, a major psychoactive opiate $[33,35]$. These results indicate that opiates (including heroin) encourage autophagy, promoting neurotoxicity via autophagic cell death in the 
striatum. In our study, the expression of LTP-related genes was also changed in the striatum of all heroin-treated groups (A1S, A2S, A3S, and A4S). Heroin addiction is associated with upregulated LTP in the NAc [36] and hippocampus [37], followed by impairments of synaptic plasticity. Based on previous studies and our current data, we speculate that heroin promotes LTP in the brain, regardless of exposure time. Further investigations are needed to determine how LTP-related genes affect synaptic plasticity in response to heroin.

In this study, circadian entrainment-related genes were differentially expressed between $\mathrm{A} 1 \mathrm{~S}$ and $\mathrm{A} 2 \mathrm{~S}$. A previous study has reported that circadian preferences are different between children and teenagers [38]. Considering that animals in groups $\mathrm{A} 1 \mathrm{~S}$ and $\mathrm{A} 2 \mathrm{~S}$ are age-matched with human children and teenagers, respectively, it is possible that the circadian rhythms of A1S and A2S are inherently different. Further studies are needed to assess whether the differential expression of these genes between A1S and $\mathrm{A} 2 \mathrm{~S}$ is due to an alteration of circadian features by heroin or just the result of age-specific circadian differences. In addition, as a result of comparing DEGs between $\mathrm{A} 2 \mathrm{~S}$ and $\mathrm{A} 4 \mathrm{~S}$, there were changes of genes associated with $\mathrm{AD}$. This implies that heroin affects the expression of genes associated with neurodegenerative disease differently in the growing brain versus the mature brain. In particular, some genes known to be upregulated during the development of $\mathrm{AD}[39,40]$ were upregulated in $\mathrm{A} 4 \mathrm{~S}$ compared to $\mathrm{A} 2 \mathrm{~S}$ (data not shown). These included Fe65/amyloid $\beta$ A4 precursor protein-binding family $\mathrm{B}$ member 1 and microtubule-associated protein tau (MAPT). These results suggest that adults may be more susceptible than teenagers to heroin-induced neurodegenerative disease.

In the present study, STMN1 was significantly downregulated in only $\mathrm{A} 2 \mathrm{H}$ group. STMN1 controls microtubule dynamics, in which the mitotic spindle assembles or disassembles in the last stage of cell division [41]. It has been reported that neurotoxicants (such as copper) cause a decrease of STMN1 in the hippocampus of the mouse, resulting in neurotoxicity [42]. Similarly, heroin may cause age-specific neurotoxicity by inhibiting the expression of STMN1 in the hippocampus of the monkey. On the contrary, FGF14 in our study decreased in all heroin-treated groups except $\mathrm{A} 2 \mathrm{H}$. FGF14 is abundant in various regions of the brain, including the hippocampus, cerebellum, and neocortex. Previous studies have reported that mutations or genetic deletions of FGF14 in animal models not only induced impairments in hippocampal synaptic plasticity and cognitive function [43], but also caused reduction of food intake and aggressivity [44]. Based on previous results and our data, the expression patterns of STMN1 and FGF14 in the $\mathrm{A} 2 \mathrm{H}$ group are opposite those of other heroin-treated groups, indicating that heroin differentially affects neurotoxicity and hippocampal synaptic plasticity depending on age. Considering that the $\mathrm{A} 2 \mathrm{H}$ group is matched with human early teenagers, a single exposure to heroin in this age group may cause neurotoxicity through different mechanisms compared to other age groups. However, as a result, even a single injection of heroin may indirectly induce impairments in hippocampal synaptic plasticity.

In this study, genes related to GABAergic synapses (GABBR2 and GAD1) were decreased in the hippocampus of all heroin-treated groups. This suggests the possibility that pre- and post-synaptic GABAergic receptors are affected by even a single injection of heroin. The GABBR2 gene encodes GABA B receptor 2 (GABABR2), which forms heterodimers with GABABR1 [45]. The GABAergic system is inhibited presynaptically by GABABRs, and alteration of the system is related to addictive behavior [46]. In our study, the $\mathrm{A} 1 \mathrm{H}$ and $\mathrm{A} 2 \mathrm{H}$ groups exhibited particularly significant decrease of GAD1 mRNA. GAD1 encodes the glutamate decarboxylase 67 protein that regulates GABA level and signaling [47]. Expression level of GAD1 is decreased in the hippocampus of patients with major depression and bipolar disorder and also in cocaine addicts $[48,49]$. Taken together, these findings imply that even a single injection of heroin negatively affects the GABAergic system, possibly promoting drug addictive behavior and psychiatric disorders.

GRIA2, a subtype of the glutamate AMPA ( $\alpha$-amino-3hydroxy-5-methyl-4-isoxazolepropionic acid) receptor, is the richest glutamate receptor in the central nervous system. It regulates excitatory neurotransmission in retrograde endocannabinoid signaling [50] and inhibits calcium influx through AMPA-receptor complexes [51]. In the present study, heroin treatment induced a decrease of GRIA2 mRNA expression in the hippocampus of all monkeys. In a study by Mead and Stephens [52], gria2 knock-out mice showed deficits in learning stimulus-reward associations. This implied that a GRIA2 deficit may negatively affect behavior, contributing to drug addiction. 
Therefore, even a single exposure to heroin is believed to decrease GRIA2 level in all ages, leading to impairments in retrograde endocannabinoid signaling.

In the present study, a single exposure of heroin caused decrease of NTRK1 and NGFR in the striatum of all animals. NTRK 1 and NGFR are genes that encode receptor proteins responsible for the development and survival of neurons [53]. In particular, NGFR was drastically decreased in all groups exposed to heroin. Nerve growth factor receptor (also known as $\mathrm{p} 75^{\mathrm{NTR}}$ ) is a low-affinity receptor that binds neurotrophins, leading to the development, survival, and function of neurons [54]. In a study by Busch et al. [54] that examined physiological and morphological changes in $p 75^{N T R}$ knockout mice, the deficiency of $p 75^{N T R}$ led to an increase of LTP in the basolateral amygdala and promoted anxiety-like behavior. In our GO analysis, we observed that LTP-related genes were changed in the striatum by heroin exposure. We can speculate that heroin may regulate LTP and negatively affect the survival of neurons in the striatum by inhibiting $p 75^{N T R}$.

We identified upregulation of $C A C N G 3$ and downregulation of NFATC4 in all heroin-treated animals. These two genes are associated with the oxytocin signaling pathway. Oxytocin signaling controls the hypothalamo-pituitary-adrenal axis through behavioral responses in the brain [55]. CACNG3 encodes the voltage-dependent calcium gamma-3 subunit that mediates trafficking to the somatodendritic compartment [56]. Little has been studied about the features or functions of $C A C N G 3$. NFATC4 increases neurogenesis and inhibits anxiety [57]. A deletion of NFATC4 causes partial defects in the encoding of hippocampal-dependent spatial memories [58]. Based on studies by Quadrato et al. [57,58] and on our results, the downregulation of NFATC4 in response to heroin may have negative effects on neurogenesis and memory function.

Autophagy is a degradation mechanism mediated by lysosomes and induced by drug addiction [59]. It is an important pathway for cells to protect against pathological factors. A previous study found that neurons exposed to morphine promote ATC5-related autophagy [60]. In this study, ATG5 gene expression increased in all herointreated groups, particularly the youngest group (A1S). ATG5-related autophagy plays a protective role in cells undergoing apoptosis [61]. Apoptotic cells exhibited an accumulation of cytoplasmic inclusion bodies in the brains of atg5-deficient mice [62]. The increase of ATG5 in the striatum may be a protective mechanism to inhibit cell death or to block the accumulation of cytoplasmic debris due to heroin. In addition, it is believed that exposure to heroin at a young age is more toxic to nerve cells, highly activating a protective mechanism.

In our study, $A K T 1$ was significantly decreased in animals of all ages exposed to heroin. The $A K T 1$ gene has a dopaminergic synapse-associated function. On the other hand, the AKT1/PKB protein kinase interaction plays an important role in cell survival [63]. Phosphorylation of AKT1 leads to its activation, which facilitates antiapoptotic action [64]. In a study by Ramos-Miguel et al. [65], chronic opiate addicts exhibited a decrease of phosphorylated AKT1 in the brain, while total AKT1 was not changed. This result suggests that chronic exposure to opiates inactivates AKT1, but does not block its expression. In contrast, our research found that acute exposure to heroin decreased $A K T 1$ expression. More studies are needed to examine whether acute exposure to heroin also affects the phosphorylation of AKT1.

In conclusion, we found that a single injection of heroin induced alterations in gene expression in the hippocampus and striatum of all tested ages of cynomolgus monkeys. When analyzing DEGs in the hippocampus, genes involved in actin cytoskeleton organization were differentially expressed in all ages of heroin-treated monkeys $(\mathrm{A} 1 \mathrm{H}, \mathrm{A} 2 \mathrm{H}, \mathrm{A} 3 \mathrm{H}$, and $\mathrm{A} 4 \mathrm{H})$ compared to the $\mathrm{ConH}$ group. This result suggests that heroin may affect a variety of cellular functions in the brain (such as motility, cellular signaling, cell adhesion, and intracellular trafficking) by reorganizing the actin cytoskeleton. When analyzing DEGs in the striatum, genes related to LTP were differentially expressed in heroin-treated groups of all ages (A1S, A2S, A3S, and A4S) compared to the ConS group. Interestingly, heroin decreased STMN1 (involved in microtubule dynamics) expression in the only hippocampus of the $\mathrm{A} 2 \mathrm{H}$ group but did not induce expression change of FGF14 only in the group. On the other hand, heroin decreased the expression of NTRK1 and NGFR (genes associated with neurotrophin signaling) in the striatum of all age groups. In particular, the amount of increase in ATG5 (involved in autophagy) expression by heroin tended to decrease. However, there were a few limitations to this study. For the RNA-Seq analyses, only one heroin-treated 
animal was used in each age group. To partially overcome this limitation, two heroin-treated animals in each age group were used to validate the results obtained from RNA-Seq and genes presenting a greater than 2-fc were selected for further analysis. To the best of our knowledge, this is the first study to analyze heroin exposure in nonhuman primates of different ages. DEG in the hippocampus and striatum were analyzed simultaneously by RNA-Seq technology.

\section{Acknowledgments}

This research was supported by the Bio \& Medical Technology Development Program of the National Research Foundation funded by the Korean government, MSIP (NRF-2014M3A9B6070246).

\section{Conflicts of Interest}

No potential conflict of interest relevant to this article was reported.

\section{Author Contributions}

Conceptualization: Mi Ran Choi, Yeung-Bae Jin, Chang-Nim Im. Experiment: Yeung-Bae Jin, Sol Hee Bang, Youngjeon Lee, Han-Na Kim, Kyu-Tae Chang. Data acquisition and analysis: Mi Ran Choi, Yeung-Bae Jin. Writing - review \& editing: Mi Ran Choi, Yeung-Bae Jin, Sang-Rae Lee, Dai-Jin Kim. Supervision and funding: Sang-Rae Lee, Dai-Jin Kim.

\section{ORCID}

Mi Ran Choi Yeung-Bae Jin https://orcid.org/0000-0001-9046-7955 Sol Hee Bang Chang-Nim Im Youngjeon Lee Han-Na Kim Kyu-Tae Chang Sang-Rae Lee Dai-Jin Kim https://orcid.org/0000-0001-6783-7970 https://orcid.org/0000-0002-8557-1026 https://orcid.org/0000-0002-7566-7075 https://orcid.org/0000-0002-5387-3441 https://orcid.org/0000-0002-0934-4411 https://orcid.org/0000-0001-5604-3657 https://orcid.org/0000-0001-8400-5973 https://orcid.org/0000-0001-9408-5639

\section{REFERENCES}

1. Alcohol and Drug Abuse Statistics. [Internet]. Brentwood: American Addiction Centers Lupdated 2019 Jun 3; cited at 2019 Jan 10]. Available from: https://americanaddictioncenters. org/rehab-guide/addiction-statistics/.

2. United Nations Office on Drugs and Crime. World Drug

Report 2015 [Internet]. New York: United Nations; 2015 May [cited at 2019 Jan 10]. Available from: https://mww.unodc.org/ documents/wdr2015/World_Drug_Report_2015.pdf.

3. Pattij T, Vanderschuren LJ. The neuropharmacology of impulsive behaviour. Trends Pharmacol Sci 2008;29:192-199.

4. Lazarov O, Hollands C. Hippocampal neurogenesis: learning to remember. Prog Neurobiol 2016;138-140:1-18.

5. Tramullas M, Martínez-Cué C, Hurlé MA. Chronic administration of heroin to mice produces up-regulation of brain apoptosis-related proteins and impairs spatial learning and memory. Neuropharmacology 2008;54:640-652.

6. Schmidt A, Walter M, Gerber H, Schmid O, Smieskova R, Bendfeldt K, et al. Inferior frontal cortex modulation with an acute dose of heroin during cognitive control. Neuropsychopharmacology 2013;38:2231-2239.

7. Zhong H, Dang J, Huo Z, Ma Z, Chen J, Huang Y, et al. Effects of medial prefrontal cortex 5-HT7 receptor knockdown on cognitive control after acute heroin administration. Brain Res 2018;1678:419-431.

8. Shahak $\mathrm{H}$, Slotkin TA, Yanai J. Alterations in PKCgamma in the mouse hippocampus after prenatal exposure to heroin: a link from cell signaling to behavioral outcome. Brain Res Dev Brain Res 2003;140:117-125.

9. Benoilid A, Collongues N, de Seze J, Blanc F. Heroin inhalation-induced unilateral complete hippocampal stroke. Neurocase 2013;19:313-315.

10. Berke JD, Hyman SE. Addiction, dopamine, and the molecular mechanisms of memory. Neuron 2000;25:515-532.

11. Marinelli M, Barrot M, Simon H, Oberlander C, Dekeyne A, Le Moal M, et al. Pharmacological stimuli decreasing nucleus accumbens dopamine can act as positive reinforcers but have a low addictive potential. EurJ Neurosci 1998;10:3269-3275.

12. Xu S, Liu Y, Li Y, Deng Y, Yuan J, Lv R, et al. Availability of dopamine transporters in heroin-dependent subjects: a 18F-FECNT PET imaging study. Psychiatry Res Neuroimaging 2017:263:121-126.

13. Egervari G, Landry J, Callens J, Fullard JF, Roussos P, Keller E, et al. Striatal H3K27 acetylation linked to g/utamatergic gene dysregulation in human heroin abusers holds promise as therapeutic target. Biol Psychiatry 2017;81:585-594.

14. Seifert CL, Magon S, Sprenger T, Lang UE, Huber CG, Denier $\mathrm{N}$, et al. Reduced volume of the nucleus accumbens in heroin addiction. Eur Arch Psychiatry Clin Neurosci 2015;265:637645.

15. Veeranagouda $Y$, Rival $P$, Prades C, Mariet C, Léonard JF, Gautier JC, et al. Identification of microRNAs in Macaca fascicularis (Cynomolgus monkey) by homology search and experimental validation by small RNA-Seq and RT-qPCR using kidney cortex tissues. PLoS One 2015;10:e0142708.

16. Weerts EM, Fantegrossi WE, Goodwin AK. The value of nonhuman primates in drug abuse research. Exp Clin Psychopharmacol 2007;15:309-327.

17. Kofler J, Lopresti B, Janssen C, Trichel AM, Masliah E, Finn OJ, 
et al. Preventive immunization of aged and juvenile non-human primates to $\beta$-amyloid. J Neuroinflammation 2012;9:84.

18. Uchida A, Sasaguri H, Kimura N, Tajiri M, Ohkubo T, Ono F, et al. Non-human primate model of amyotrophic lateral sclerosis with cytoplasmic mislocalization of TDP-43. Brain 2012; 135(Pt 3):833-846.

19. GresI TA, Colman RJ, Roecker EB, Havighurst TC, Huang Z, Allison DB, et al. Dietary restriction and glucose regulation in aging rhesus monkeys: a follow-up report at $8.5 \mathrm{yr}$. Am J Physiol Endocrinol Metab 2001;281:E757-E765.

20. Choi MR, Bang SH, Jin YB, Lee Y, Kim HN, Chang KT, et al. Effects of methamphetamine in the hippocampus of Cynomolgus monkeys according to age. BioChip J 2017;11:272-285.

21. Evans SM, Nasser J, Comer SD, Foltin RW. Smoked heroin in rhesus monkeys: effects of heroin extinction and fluid availability on measures of heroin seeking. Pharmacol Biochem Behav 2003;74:723-737.

22. Bolger AM, Lohse M, Usadel B. Trimmomatic: a flexible trimmer for Illumina sequence data. Bioinformatics 2014;30: 2114-2120.

23. Trapnell C, Williams BA, Pertea G, Mortazavi A, Kwan G, van Baren MJ, et al. Transcript assembly and quantification by $R N A$-Seq reveals unannotated transcripts and isoform switching during cell differentiation. Nat Biotechnol 2010;28:511515.

24. Giulietti A, Overbergh L, Valckx D, Decallonne B, Bouillon R, Mathieu C. An overview of real-time quantitative PCR: applications to quantify cytokine gene expression. Methods 2001; 25:386-401.

25. Elzagallaai AA, Greff M, Rieder MJ. Adverse drug reactions in children: the double-edged sword of therapeutics. Clin Pharmacol Ther 2017;101:725-735.

26. Laviola G, Adriani W, Terranova ML, Gerra G. Psychobiological risk factors for vulnerability to psychostimulants in human adolescents and animal models. Neurosci Biobehav Rev 1999;23:993-1010.

27. Schultz W, Tremblay L, Hollerman JR. Reward prediction in primate basal ganglia and frontal cortex. Neuropharmacology 1998;37:421-429.

28. Wang D, Chadha GK, Feygin A, Ivanov Al. F-actin binding protein, anillin, regulates integrity of intercellular junctions in human epithelial cells. Cell Mol Life Sci 2015;72:3185-3200.

29. Lovinger DM. Presynaptic modulation by endocannabinoids. Handb Exp Pharmacol 2008;(184):435-477.

30. Fourgeaud L, Mato S, Bouchet D, Hémar A, Worley PF, Manzoni OJ. A single in vivo exposure to cocaine abolishes endocannabinoid-mediated long-term depression in the nucleus accumbens. J Neurosci 2004;24:6939-6945.

31. Mah A, Geeraert B, Lebel C. Detailing neuroanatomical development in late childhood and early adolescence using NODDI. PLoS One 2017;12:e0182340.

32. Glick D, Barth S, Macleod KF. Autophagy: cellular and molecular mechanisms. J Pathol 2010;221:3-12.
33. Feng YM, Jia YF, Su LY, Wang D, Lv L, Xu L, et al. Decreased mitochondrial DNA copy number in the hippocampus and peripheral blood during opiate addiction is mediated by autophagy and can be salvaged by melatonin. Autophagy 2013;9:1395-1406.

34. Kovacs GG, Horvath MC, Majtenyi K, Lutz MI, Hurd YL, Keller E. Heroin abuse exaggerates age-related deposition of hyperphosphorylated tau and p62-positive inclusions. Neurobiol Aging 2015;36:3100-3107.

35. Zhao L, Zhu Y, Wang D, Chen M, Gao P, Xiao W, et al. Morphine induces Beclin 1- and ATG5-dependent autophagy in human neuroblastoma SH-SY5Y cells and in the rat hippocampus. Autophagy 2010;6:386-394.

36. Shen H, Moussawi K, Zhou W, Toda S, Kalivas PW. Heroin relapse requires long-term potentiation-like plasticity mediated by NMDA2b-containing receptors. Proc Natl Acad Sci US A 2011;108:19407-19412.

37. Bao G, Kang L, Li H, Li Y, Pu L, Xia P, et al. Morphine and heroin differentially modulate in vivo hippocampal LTP in opiate-dependent rat. Neuropsychopharmacology 2007;32: 1738-1749.

38. Adan A, Archer SN, Hidalgo MP, Di Milia L, Natale V, Randler C. Circadian typology: a comprehensive review. Chronobiol Int 2012;29:1153-1175.

39. Bejanin A, Schonhaut DR, La Joie R, Kramer JH, Baker SL, Sosa $\mathrm{N}$, et al. Tau pathology and neurodegeneration contribute to cognitive impairment in Alzheimer's disease. Brain 2017; 140:3286-3300.

40. Csiszar A, Tucsek Z, Toth P, Sosnowska D, Gautam T, Koller $A$, et al. Synergistic effects of hypertension and aging on cognitive function and hippocampal expression of genes involved in $\beta$-amyloid generation and Alzheimer's disease. Am J Physiol Heart Circ Physiol 2013;305:H1120-H1130.

41. Jeon TY, Han ME, Lee YW, Lee YS, Kim GH, Song GA, et al. Overexpression of stathmin 1 in the diffuse type of gastric cancer and its roles in proliferation and migration of gastric cancer cells. Br J Cancer 2010;102:710-718.

42. Yu H, Jiang X, Lin X, Zhang Z, Wu D, Zhou L, et al. Hippocampal subcellular organelle proteomic alteration of copper-treated mice. Toxicol Sci 2018;164:250-263.

43. Xiao M, Xu L, Laezza F, Yamada K, Feng S, Ornitz DM. Impaired hippocampal synaptic transmission and plasticity in mice lacking fibroblast growth factor 14. Mol Cell Neurosci 2007; 34:366-377.

44. Hoxha E, Marcinnò A, Montarolo F, Masante L, Balbo I, Ravera $\mathrm{F}$, et al. Emerging roles of Fgf14 in behavioral control. Behav Brain Res 2019;356:257-265.

45. Bettler B, Kaupmann K, Mosbacher J, Gassmann M. Molecular structure and physiological functions of GABA(B) receptors. Physiol Rev 2004;84:835-867.

46. Le Moal M, Koob GF. Drug addiction: pathways to the disease and pathophysiological perspectives. Eur Neuropsychopharmacol 2007;17:377-393. 
47. Varju P, Katarova Z, Madarász E, Szabó G. Sequential induction of embryonic and adult forms of glutamic acid decarboxylase during in vitro-induced neurogenesis in cloned neuroectodermal cell-line, NE-7C2. I Neurochem 2002;80: 605-615.

48. Enoch MA, Zhou Z, Kimura M, Mash DC, Yuan Q, Goldman D. GABAergic gene expression in postmortem hippocampus from alcoholics and cocaine addicts; corresponding findings in alcohol-naïve $P$ and NP rats. PLoS One 2012;7:e29369.

49. Tao R, Davis KN, Li C, Shin JH, Gao Y, Jaffe AE, et al. GAD1 alternative transcripts and DNA methylation in human prefrontal cortex and hippocampus in brain development, schizophrenia. Mol Psychiatry 2017;23:1496-1505.

50. El Manira A, Kyriakatos A, Nanou E, Mahmood R. Endocannabinoid signaling in the spinal locomotor circuitry. Brain Res Rev 2008;57:29-36.

51. Jia Z, Agopyan N, Miu P, Xiong Z, Henderson J, Gerlai R, et al. Enhanced LTP in mice deficient in the AMPA receptor GluR2. Neuron 1996;17:945-956.

52. Mead AN, Stephens DN. Involvement of AMPA receptor GluR2 subunits in stimulus-reward learning: evidence from glutamate receptor gria2 knock-out mice. I Neurosci 2003; 23:9500-9507.

53. Kerr B, Garcia-Rudaz C, Dorfman M, Paredes A, Ojeda SR. NTRK1 and NTRK2 receptors facilitate follicle assembly and early follicular development in the mouse ovary. Reproduction 2009;138:131-140.

54. Busch R, Baldus M, Vogt MA, Berger SM, Bartsch D, Gass P, et al. Effects of $p 75$ NTR deficiency on cholinergic innervation of the amygdala and anxiety-like behavior. I Neurochem 2017;141:461-471.

55. Neumann ID. Involvement of the brain oxytocin system in stress coping: interactions with the hypothalamo-pituitary-adrenal axis. Prog Brain Res 2002;139:147-162.

56. Moss FJ, Dolphin AC, Clare JJ. Human neuronal stargazin-like proteins, gamma2, gamma3 and gamma4; an investigation of their specific localization in human brain and their influence on CaV2.1 voltage-dependent calcium channels expressed in
Xenopus oocytes. BMC Neurosci 2003;4:23.

57. Quadrato G, Elnaggar MY, Duman C, Sabino A, Forsberg K, Di Giovanni S. Modulation of GABAA receptor signaling increases neurogenesis and suppresses anxiety through NFATC4. J Neurosci 2014;34:8630-8645.

58. Quadrato G, Benevento M, Alber S, Jacob C, Floriddia EM, Nguyen T, et al. Nuclear factor of activated T cells (NFATC4) is required for BDNF-dependent survival of adult-born neurons and spatial memory formation in the hippocampus. Proc Natl Acad Sci U S A 2012;109:E1499-E1508.

59. Levine B, Yuan J. Autophagy in cell death: an innocent convict? J Clin Invest 2005; 115:2679-2688.

60. Su LY, Luo R, Liu Q, Su JR, Yang LX, Ding YQ, et al. Atg5- and Atg7-dependent autophagy in dopaminergic neurons regulates cellular and behavioral responses to morphine. Autophagy 2017;13:1496-1511.

61. Garcia-Garcia A, Anandhan A, Burns M, Chen H, Zhou Y, Franco R. Impairment of Atg5-dependent autophagic flux promotes paraquat- and $\mathrm{MPP}^{+}$-induced apoptosis but not rotenone or 6-hydroxydopamine toxicity. Toxicol Sci 2013; 136:166-182.

62. Hara T, Nakamura K, Matsui M, Yamamoto A, Nakahara Y, Suzuki-Migishima R, et al. Suppression of basal autophagy in neural cells causes neurodegenerative disease in mice. Nature 2006;441:885-889.

63. Song G, Ouyang G, Bao S. The activation of Akt/PKB signaling pathway and cell survival. I Cell Mol Med 2005;9:59-71.

64. Trencia A, Perfetti A, Cassese A, Vigliotta G, Miele C, Oriente $\mathrm{F}$, et al. Protein kinase B/Akt binds and phosphorylates PED/PEA-15, stabilizing its antiapoptotic action. Mol Cell Biol 2003;23:4511-4521.

65. Ramos-Miguel A, García-Fuster MJ, Callado LF, La Harpe R, Meana JJ, García-Sevilla JA. Phosphorylation of FADD (Fasassociated death domain protein) at serine 194 is increased in the prefrontal cortex of opiate abusers: relation to mitogen activated protein kinase, phosphoprotein enriched in astrocytes of $15 \mathrm{kDa}$, and Akt signaling pathways involved in neuroplasticity. Neuroscience 2009;161:23-38. 